1997

\title{
Frequency dependent effects in helicon plasmas
}

Paul A. Keiter

Earl E. Scime

Matthew M. Balkey

Follow this and additional works at: https://researchrepository.wvu.edu/faculty_publications

\section{Digital Commons Citation}

Keiter, Paul A.; Scime, Earl E.; and Balkey, Matthew M., "Frequency dependent effects in helicon plasmas" (1997). Faculty Scholarship. 174.

https://researchrepository.wvu.edu/faculty_publications/174 


\title{
Frequency dependent effects in helicon plasmas
}

\author{
Paul A. Keiter, Earl E. Scime, and Matthew M. Balkey \\ Department of Physics, West Virginia University, Morgantown, West Virginia 26506
}

(Received 26 November 1996; accepted 16 April 1997)

\begin{abstract}
Variations in the plasma parameters of a large volume, helicon source as a function of applied rf power $(0-2 \mathrm{~kW})$, driving frequency $(8-18 \mathrm{MHz})$, magnetic field $(0-1.4 \mathrm{kG})$ and fill pressure $(2-10$ mTorr) have been studied. The transitions between the capacitive, inductive, and resonant helicon mode are consistent with previous experiments. Our data indicate that the transition to the helicon mode occurs at a unique magnetic field, independent of the driving frequency. Based on the helicon wave dispersion relation, from which helicon wavelengths can be calculated, the observed variations in plasma density as a function of driving frequency suggest that the wavelength of the helicon wave is a weak function of driving frequency. Calculation of the electron energies which correspond to the phase velocity of the driving wave (i.e., Landau damping) suggest that either Landau damping cannot be responsible for the efficient ionization of helicon sources, or that the helicon portion of the discharge does not extend over the entire radius of the apparatus. (C) 1997 American Institute of Physics. [S1070-664X(97)04307-3]
\end{abstract}

\section{INTRODUCTION}

Since Boswell's helicon wave experiments in the 1970s demonstrated efficient production of high density plasmas with a few kilowatts of rf power, ${ }^{1-3}$ a variety of experiments have been undertaken to investigate the physics of helicon discharges. The early experiments of Boswell and coworkers clarified the dependence of helicon plasma density on magnetic field strength, chamber size and $\mathrm{rf}$ power. ${ }^{2}$ Later researchers provided detailed measurements of the densities, temperatures, and magnetic fields within helicon sources as a function of $\mathrm{rf}$ power, antenna configuration, magnetic field strength, and neutral pressure. ${ }^{4-11}$ Those measurements were in good agreement with theoretical predictions. ${ }^{7,8,12-14}$

The helicon wave, from which the helicon source derives its name, is a variant of the classic right-hand, circularly polarized electromagnetic wave-the whistler wave. In the case of helicon discharges, the wave is confined inside an insulating cylinder and generally lies in the frequency range $\omega_{c i} \ll\left(\omega_{c i} \omega_{c e}\right)^{1 / 2} \ll \omega \ll \omega_{c e} \ll \omega_{p e} .{ }^{15}$ The insulating boundary adds additional constraints to the wave dispersion relation. Theoretical and experimental investigations of helicon discharges have tended to focus on two issues: the mode structure of the helicon wave and the mechanism responsible for the efficient ionization of the plasma in the helicon source.

For predicting the helicon wave mode structure, the dispersion relation for a helicon wave in a plasma bounded by an insulating cylinder can be derived analytically. Chen ${ }^{16}$ has derived such a dispersion relation for the case of a nonuniform plasma and discussed the impact of effects such as electron inertia and wave polarization. ${ }^{17} \mathrm{~A}$ number of researchers have measured the spatial dependence of the wave magnetic fields in helicon sources. ${ }^{7,8,12-14}$ For the type of antenna used in these experiments, a Nagoya type III, ${ }^{18}$ typical wave magnetic field measurements are consistent with theoretical predictions for a plasma dominated by the lowest azimuthal mode $(m=+1)$ and lowest radial mode. ${ }^{12,13} \mathrm{Su}-$ perposition of multiple azimuthal and radial modes has also been observed in helicon experiments. ${ }^{12,14}$

In cases of low densities, i.e., below $10^{13} \mathrm{~cm}^{-3}$, Landau damping has been suggested as the dominant mechanism for energy transfer from the wave to the plasma. ${ }^{17}$ At higher densities, collisional damping appears to be sufficient to explain the observed plasma ionization. ${ }^{9,19}$ Alternative mechanisms have been suggested such as ion cyclotron absorption, ${ }^{19}$ but wave phase velocity measurements appear to support the Landau damping hypothesis in low density helicon plasmas. ${ }^{4,7,9}$

In this paper, we present initial results from a new helicon experiment. Although other helicon experiments have operated at a variety of driving frequencies (e.g., 7, 8, 13.56, and $27.12 \mathrm{MHz}$ ) and one group has reported results for two different frequencies within the same device, ${ }^{12}$ the experiment described here is unique in that the driving frequency is continuously variable from 0.3 to $35 \mathrm{MHz}$. For the experiments reported in this paper, the driving frequency was varied from 8 to $20 \mathrm{MHz}$ because of limitations in the antenna impedance matching circuitry. In Sec. II we describe the experimental apparatus, diagnostics, and the characteristics of a typical plasma. In Sec. III we present plasma measurements as function of driving frequency for different magnetic fields. In Sec. IV, we review the relevant theory and discuss the frequency dependence of the plasma parameters in terms of the phase velocity of the helicon wave and the corresponding resonant electron energy.

\section{SOURCE PARAMETERS, DIAGNOSTICS, AND PLASMA CHARACTERISTICS}

Our steady-state helicon source [Fig. 1(a)] consists of a $15.2 \mathrm{~cm}$ inner diameter, $157 \mathrm{~cm}$ long Pyrex tube and a $19 \mathrm{~cm}$ long, Nagoya Type III antenna made from $2 \mathrm{~cm}$ wide copper strips. The antenna is driven with up to $2 \mathrm{~kW}$ of rf power over a frequency range of $0.3-35 \mathrm{MHz}$. Impedance matching for frequencies from $8-20 \mathrm{MHz}$ is accomplished with a standard capacitive Pi circuit. Impedance matching over the full range of $0.3-35 \mathrm{MHz}$ range will require additional capaci- 

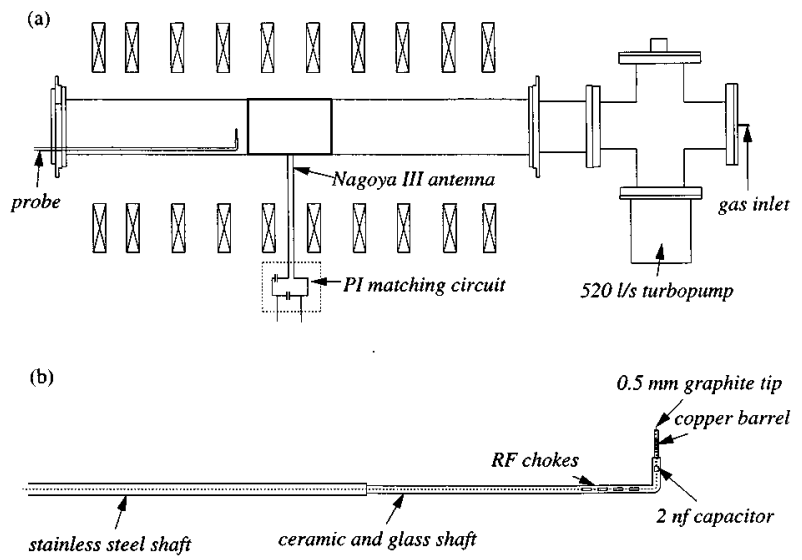

FIG. 1. (a) Schematic of helicon source with $19 \mathrm{~cm}$ long, Nagoya III antenna. (b) Schematic of rf compensated Langmuir probe used for electron density and temperature measurements. Copper barrel coupled to tip enables tip to follow floating potential fluctuations (Ref. 20).

tors. Typically, argon gas pressures of 3-10 mTorr are used. Preliminary measurements of helium plasma parameters (central density and electron temperature) at a driving frequency of $10 \mathrm{MHz}$ showed no significant differences from the argon results. The magnetic field geometry was a simple solenoid and the field strength is variable from 0 to $1.4 \mathrm{kG}$. The magnetic field strength varies by less than $0.5 \%$ across the entire plasma radius and less than $1 \%$ over the central half of the plasma (Fig. 2).

Electron densities and temperatures are measured with rf compensated Langmuir probes [Fig. 1(b)]. ${ }^{20}$ The probes are calibrated with a $9.25 \mathrm{GHz}$ microwave reflectometry system ${ }^{21}$ for density measurements. The electron temperature measurements are somewhat sensitive to the choice of $\mathrm{rf}$ compensation method. The Langmuir probe density measurements are extremely reproducible, but our relatively high electron temperature values in the helicon mode compared to other helicon experiments suggest that at high rf power we may be overestimating the electron temperature by as much as a factor of 2. This would result in an underestimation of the electron density by a factor of 2 .

The rf power scan at a driving frequency of $10 \mathrm{MHz}$ and a magnetic field of $0.5 \mathrm{kG}$ displayed in Fig. 3(a) shows the classic signature of a transition between the capacitive, in-

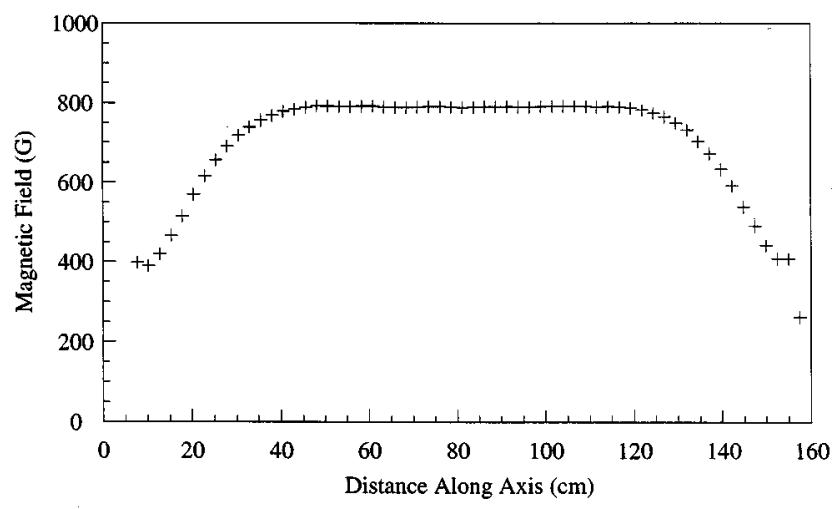

FIG. 2. Magnetic field along axis of experiment.
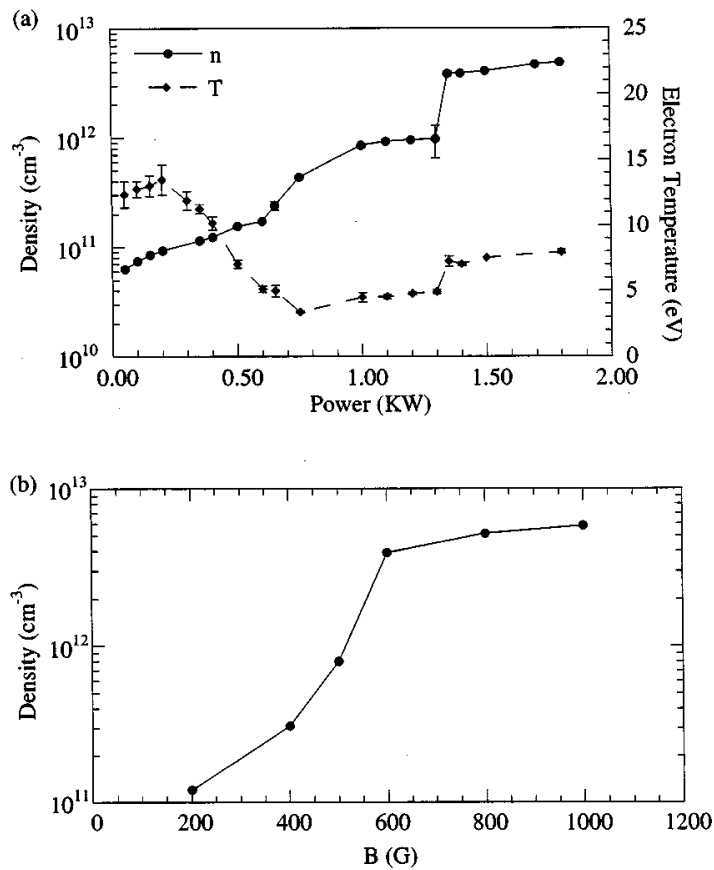

FIG. 3. (a) Central electron density and electron temperature versus rf power at $10 \mathrm{MHz}$ and $500 \mathrm{G}$. The capacitive to inductive and inductive to helicon transitions are clearly visible. Error bars indicate measurement reproducibility. (b) Central electron density versus magnetic field strength at $10 \mathrm{MHz}$ and an rf power of $1 \mathrm{~kW}$.

ductive, and helicon modes of operation. ${ }^{8}$ As the magnetic field is increased for a fixed rf power and frequency, only the transition from the capacitive to helicon mode is evident [Fig. 3(b)]. Once the helicon resonance is reached, the $\mathrm{rf}$ power can often be lowered $30 \%$ to $40 \%$ without loss of the helicon mode. All power scans reported here start from low power and increase monotonically to avoid this hysteresis effect.

As seen in other helicon experiments, there is a threshold neutral pressure for sustaining the high density helicon mode. ${ }^{22}$ Above the threshold, the change in the plasma density with increasing neutral pressure [Fig. 4(a)] is insignificant. Although the $800 \mathrm{G}$ measurements have a slightly higher density, consistent with a pattern of increased density with stronger magnetic fields, both the 600 and $800 \mathrm{G}$ pressure scans exhibit the same trend.

A radial profile for a typical plasma in the helicon mode is shown in Fig. 4(b). The density and electron temperatures vary by approximately $50 \%$ over the central half of the plasma column. Except in the region near the grounded endplate, the density profile is relatively independent of distance from the antenna [Fig. 4(c)]. The density profile does become more peaked with increasing rf power and magnetic field (as seen in other experiments ${ }^{11}$ ), but there is no significant difference in profile between the 10 and $11 \mathrm{MHz}$ cases [Fig. 4(d)].

\section{PLASMA PARAMETERS AS A FUNCTION OF DRIVING FREQUENCY}

The most comprehensive measurements were performed at a driving frequency of $10 \mathrm{MHz}$ and a fill pressure of 6 

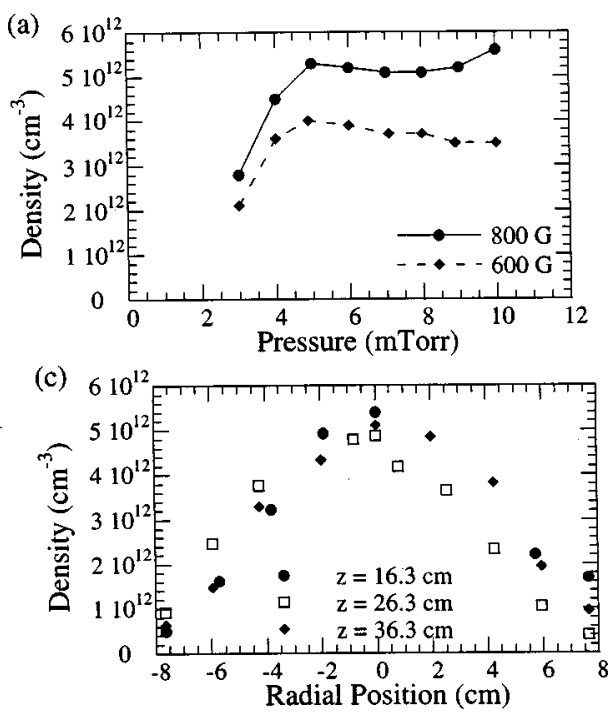
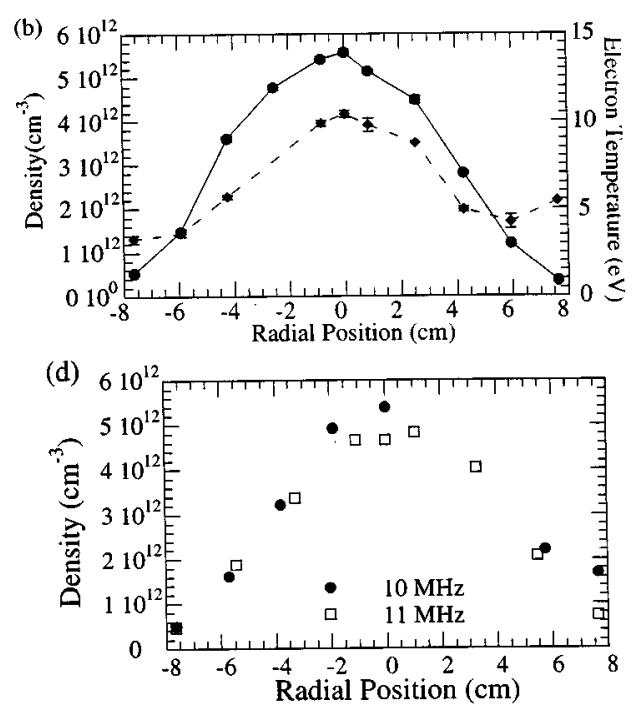

FIG. 4. (a) Electron density versus pressure for 600 and $800 \mathrm{G}$ at $10 \mathrm{MHz}$ and $1.0 \mathrm{~kW}$. (b) Radial profile of electron density (solid line) and temperature (dashed line) for a helicon mode plasma at $11 \mathrm{MHz}, 800 \mathrm{G}, 1.4 \mathrm{~kW}$, and 6 mTorr. (c) Radial profile of electron density along magnetic field at 16.3 , 26.3, and $36.3 \mathrm{~cm}$ away from the antenna at $600 \mathrm{G}$ and $1.4 \mathrm{~kW}$. (d) Radial profile of electron density for $600 \mathrm{G}$ and $1.4 \mathrm{~kW}$ at 10 and $11 \mathrm{MHz}$.

mTorr. The plasma density versus rf power for six different magnetic field strengths is shown in Fig. 5. Even with $2 \mathrm{~kW}$ of rf power, the helicon mode was not reached at 200 or 400 $\mathrm{G}$. The helicon mode was obtained for 500, 600, 800, and $1000 \mathrm{G}$, but at progressively lower rf powers for larger magnetic field strengths. At $500 \mathrm{G}$, the helicon mode was achieved at an rf power of $1.4 \mathrm{~kW}$. At $600 \mathrm{G}$ only $0.75 \mathrm{~kW}$ was required and at $800 \mathrm{G}$, less than $0.5 \mathrm{~kW}$ of $\mathrm{rf}$ power was needed. Although there is a sixfold difference in the power threshold among the various magnetic field strengths, after reaching the helicon mode the final densities vary by less than $30 \%$. At $1.4 \mathrm{~kW}$, the density ranges from 3.9 $\times 10^{12} \mathrm{~cm}^{-3}(500 \mathrm{G})$ to $5.7 \times 10^{12} \mathrm{~cm}^{-3}(800 \mathrm{G})$.

Density versus rf power for three different magnetic fields at a driving frequency of $11 \mathrm{MHz}$ is shown in Fig. 6. Consistent with the $10 \mathrm{MHz}$ data, as the magnetic field is increased, the power threshold needed to enter the helicon mode decreases. At 500, 600, and $800 \mathrm{G}, 1.6,1$, and $0.6 \mathrm{~kW}$ are needed, respectively, to reach the helicon mode. Based

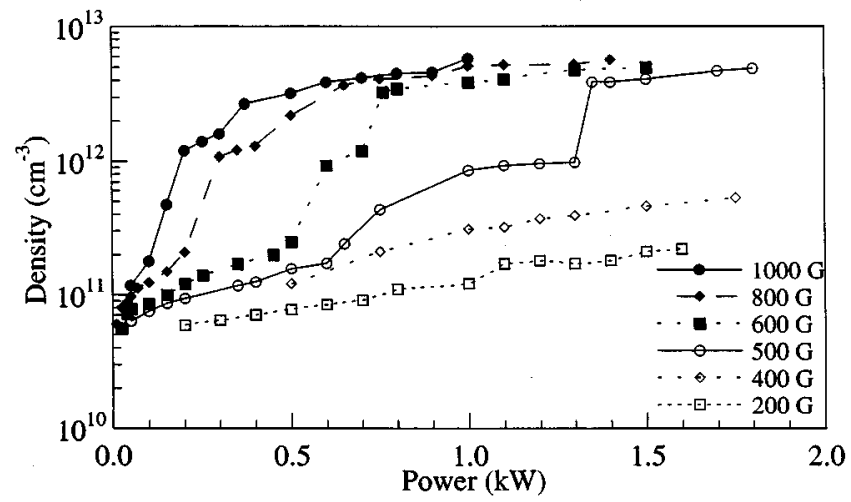

FIG. 5. Electron density versus rf power for six different magnetic fields at a driving frequency of $10 \mathrm{MHz}$ and a fill pressure of $6 \mathrm{mTorr}$. on Figs. 5 and 6, it can be concluded that less power is required to make the helicon transition at $10 \mathrm{MHz}$ than 11 $\mathrm{MHz}$ for the same magnetic field strengths.

Figure 7 displays the electron density versus rf power for five different driving frequencies at a fixed magnetic field strength. As the driving frequency of the antenna is increased, the power needed to reach the helicon mode also increases. We see that helicon plasmas at $10 \mathrm{MHz}$ require about half the power and achieve twice the electron density of $13 \mathrm{MHz}$ helicon plasmas. The data indicate that as the driving frequency is decreased, the threshold power for the helicon mode decreases and the electron density increases.

Assuming a small aspect ratio source (long with a small radius), $\mathrm{Chen}^{17}$ derived a simple version of the helicon wave dispersion relation,

$$
n=\frac{k_{\perp} \alpha B_{0}}{\mu_{0} e}\left(\frac{k_{\|}}{\omega}\right),
$$

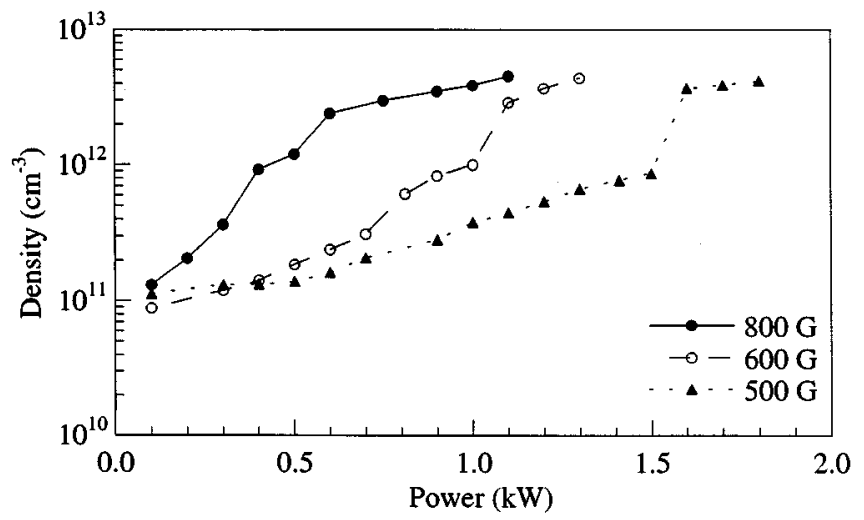

FIG. 6. Electron density versus rf power for three different magnetic fields and a driving frequency of $11 \mathrm{MHz}$. 


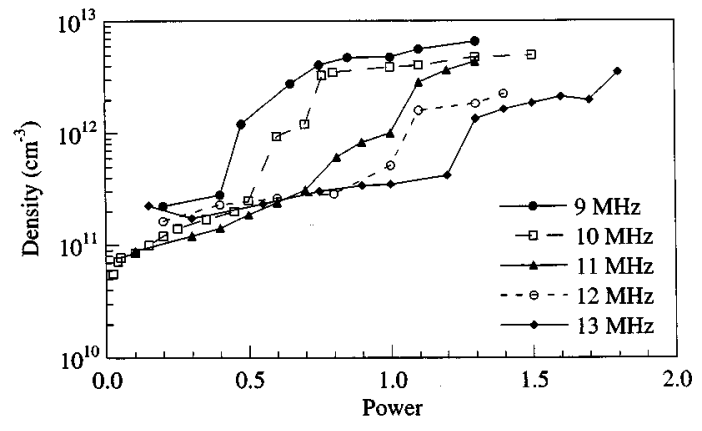

FIG. 7. Electron density versus rf power for five different rf driving frequencies at a magnetic field of $600 \mathrm{G}$.

where $\alpha^{2} \equiv k_{\perp}^{2}+k_{\|}^{2}$. He then calculated the optimum driving frequency for helicon sources with different radial dimensions. The optimum frequency was chosen by setting the phase velocity of the helicon wave equal to the optimum energy for argon ionization by electron collisions, $50 \mathrm{eV} .{ }^{17}$ This assumes that the helicon wave experiences Landau damping and creates an enhanced population of energetic electrons with energy of $50 \mathrm{eV}$. The idea is that the energetic electrons are then responsible for the high ionization efficiency of the helicon source. The small aspect ratio approximation permits the use of a single perpendicular wave number for the helicon wave at all frequencies.

Figure 8 summarizes the frequency dependence of the electron density associated with the helicon mode for three different magnetic fields. In the low-field case of $600 \mathrm{G}$ [Fig. $8(\mathrm{a})]$, below $1 / f=0.0952 \times 10^{-6} \mathrm{~s}(f=10.5 \mathrm{MHz})$ the $\mathrm{rf}$ power is insufficient to maintain the helicon mode and the density decreases dramatically. In Fig. 8(a), the data corre-


FIG. 8. (a) Electron density versus driving frequency for $600 \mathrm{G}$. The transition from the inductive to the helicon mode is seen at about $11 \mathrm{MHz}$. (b) Electron density versus driving frequency for $800 \mathrm{G}$ (circles) and $1000 \mathrm{G}$ (squares). Rf power was $1.0 \mathrm{~kW}$ for both graphs.

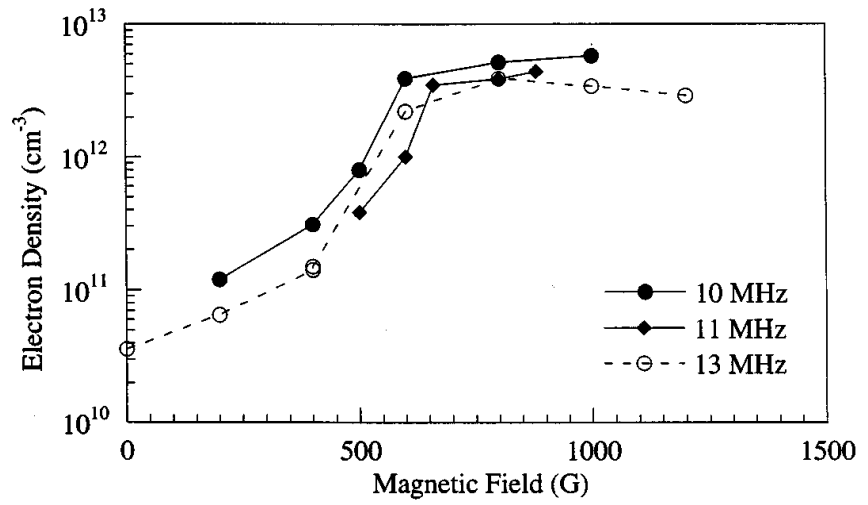

FIG. 9. Electron density versus magnetic field for three different driving frequencies. The 10 and $11 \mathrm{MHz}$ data were taken at $1 \mathrm{~kW}$. The $13 \mathrm{MHz}$ data were taken at $1.4 \mathrm{~kW}$.

sponding to the helicon mode (filled circles) are consistent with the simple helicon dispersion relation since the electron density is inversely dependent on the driving frequency. According to Eq. (1), the value of $k_{\perp} k_{\|} \alpha$ must also vary linearly with frequency in order to maintain the linear relationship between density and frequency. At higher magnetic fields, the electron density is still inversely dependent on driving frequency, but with a different slope [Fig. 8(b)]. The different slopes suggest that the resonant wavelength depends on the magnetic field for a fixed driving frequency and fixed antenna length.

Figure 9 shows the helicon mode threshold as a function of magnetic field for three different driving frequencies. Note that the minimum magnetic field strength required to initiate the helicon mode is relatively independent of driving frequency: the threshold magnetic field is approximately $500 \mathrm{G}$ in all three cases. Apparently, if the available power is sufficient to excite the helicon mode, the conditions for the helicon resonance are determined by the boundary conditions (size of chamber and antenna geometry) and the magnetic field magnitude, but not the driving frequency. Together, Figs. 8 and 9 suggest that a critical magnetic field strength is needed to "fit" the helicon wave into the chamber.

The inverse dependence of electron density with frequency is consistent with the dispersion relation for a bounded helicon wave. However, the data can also be used to investigate the nature of the physical mechanism responsible for the efficient ionization of helicon sources. Since the helicon dispersion relation defines a unique wavelength for a given plasma density and magnetic field, the measurements at different frequencies can be used to calculate the helicon wave's parallel phase velocity and expected resonant electron energy assuming a Landau damping energy transfer process $\left(E_{\mathrm{res}}=0.5 m_{e} v_{p}^{2}\right)$. As suggested by Chen, ${ }^{17}$ the resonant energy should lie near or above $50 \mathrm{eV}$, the optimum energy for electron impact ionization of argon. If the resonant energy falls below $50 \mathrm{eV}$, one might expect a density decrease for the same amount of input power. If the resonant energy falls below the $15.8 \mathrm{eV}$ ionization energy of argon, it seems unlikely that Landau damping could be responsible for the high ionization efficiency of helicon sources. For a small 
aspect ratio source, $\mathrm{Chen}^{4}$ showed that the resonant energy is given by

$$
E_{\text {res }}(\mathrm{eV})=\left(\frac{32.1 B}{a n}-7.2 a f\right)^{2},
$$

where $a$ is in $\mathrm{cm}, B$ is in $\mathrm{kG}, n$ is $10^{13} \mathrm{~cm}^{-3}$, and $f$ is in $\mathrm{GHz}$ (this equation in Ref. 4 is missing a factor of $a$ in the second term and the exponent on the sum). For $a=7.56 \mathrm{~cm}, B$ $=0.8 \mathrm{kG}, n=0.49 \times 10^{13} \mathrm{~cm}^{-3}$, and $f=10 \mathrm{MHz}$, Eq. (2) yields $E_{\text {res }}=41 \mathrm{eV}$, which is well below the optimum energy for ionization of $50 \mathrm{eV}$ for argon. The data, however, show no sign of decreasing ionization efficiency. At $f$ $=8.25 \mathrm{MHz}\left(n=0.66 \times 10^{13} \mathrm{~cm}^{-3}\right)$ the resonant energy according to Eq. (2) drops to $22.4 \mathrm{eV}$, yet the density increases by nearly $40 \%$ and the helicon power threshold drops as well. At first glance, these measurements appear to indicate that the resonant energy for an enhanced population of electrons is irrelevant. Thus Landau damping would seem to be eliminated as a possible electron energization mechanism.

However, Eq. (2) is based on the assumption of a small aspect ratio and that the source radius determines the boundary conditions for the helicon dispersion relationship. If a smaller radius is used, the resonant energy determined by Eq. (2) can be significantly larger, e.g., at the $8.25 \mathrm{MHz}$ parameters, $E_{\text {res }}=56 \mathrm{eV}$ for $a=5 \mathrm{~cm}$. Visual observations of the plasma suggest that the helicon wave may only exist over a smaller radius-a bright core of plasma of $a \approx 5 \mathrm{~cm}$ is observed when the source is in the helicon mode. To more fully examine the effects of boundary conditions and the source radius, the full, bounded helicon dispersion equation for an uniform plasma is developed in the next section.

\section{COMPLETE DISPERSION RELATIONSHIP CALCULATION}

As shown by Chen, ${ }^{17}$ for a helicon wave of the form $\exp [(i(m \theta+k z-\omega t)]$ in a cylindrical plasma with an axial magnetic field, Maxwell's equations for the wave quantities lead to the following equations for the wave magnetic field components:

$$
\begin{aligned}
& B_{z}^{\prime \prime}+f(r) B_{z}^{\prime}+g(r) B_{z}=0, \\
& k_{\perp}^{2} B_{r}=\frac{i m \alpha}{r} B_{z}+i k_{\|} \gamma B_{z}^{\prime}, \\
& k_{\perp}^{2} B_{\theta}=-\alpha B_{z}^{\prime}-\frac{m k_{\|} \gamma}{r} B_{z},
\end{aligned}
$$

where ${ }^{\prime}=\partial / \partial r$ and

$$
\begin{aligned}
& f(r)=\frac{1}{r}-\frac{2 \alpha \alpha^{\prime}}{k_{\perp}^{2}}, \\
& g(r)=\frac{k_{\perp}^{2}}{\gamma}-\frac{m^{2}}{r^{2}}-\frac{m \alpha^{\prime}}{k_{\|} \gamma r}\left(1+\frac{2 k_{\|}^{2} \gamma^{2}}{k_{\perp}^{2}}\right),
\end{aligned}
$$

and

$$
\alpha(r)=\frac{\omega \mu_{0} e}{B_{0} k_{\|}} n(r), \quad \gamma=1-\left(\frac{\omega}{c k_{\|}}\right)^{2}, \quad k_{\perp}^{2}=\alpha^{2}-k_{\|}^{2} .
$$

Because experiments have shown that Nagoya III antennas predominately generate the $m=+1$ mode and the $m=+1$ mode structure predictions assuming a uniform plasma are consistent with the experimental data, ${ }^{12,13}$ we will assume that the uniform plasma limit is sufficient to model our plasma. We also assume that the displacement current in the plasma can be neglected $\left(\omega / c k_{\|} \ll 1\right)$. Thus

$$
B_{z}^{\prime \prime}+\frac{1}{r} B_{z}^{\prime}+\left(k_{\perp}^{2}-\frac{m^{2}}{r^{2}}\right) B_{z}=0
$$

where

$$
k_{\perp}^{2}=\left(\frac{\omega \mu_{0} e}{B_{0} k_{\|}} n\right)^{2}-k_{\|}^{2} .
$$

The solutions of Eq. (9) are the integer-order Bessel functions $J_{m}\left(k_{\perp} r\right)$. The boundary condition on the current at the insulating wall of the plasma, $j_{r}(a) \propto B_{r}(a)=0$, places a constraint on Eq. (4) at $r=a$

$$
0=\frac{m \alpha}{a} J_{m}\left(k_{\perp} a\right)+k_{\|} J_{m}^{\prime}\left(k_{\perp} a\right) .
$$

For $m=0$, Eq. (11) becomes

$$
0=J_{1}\left(k_{\perp} a\right),
$$

and each root of the first Bessel function corresponds to a different radial mode of the $m=0$ helicon wave $(n=1,2,3$, etc.). Then Eq. (10) can be solved for $k_{\|}$. Assuming a small aspect ratio, this is the same result as Eq. (1) since the first root of the first Bessel function is 3.83. For the more general $m=1$ case of interest here,

$$
0=\left(\frac{\omega \mu_{0} e}{a B_{0}} n_{0}\right) J_{1}\left(k_{\perp} a\right)+k_{\|}^{2} J_{1}^{\prime}\left(k_{\perp} a\right),
$$

must be solved to determine $k_{\perp}$. Using Eq. (10) to replace $k_{\|}$, yields

$$
\begin{aligned}
0= & \left(\frac{\omega \mu_{0} e}{a B_{0}} n_{0}\right) J_{1}\left(k_{\perp} a\right) \\
& +\left(\frac{\left.-k_{\perp}^{2}+\sqrt{k_{\perp}^{4}+4\left(\frac{\omega \mu_{0} e}{B_{0}} n_{0}\right)^{2}}\right)}{2}\right) J_{1}^{\prime}\left(k_{\perp} a\right),
\end{aligned}
$$

which can be solved numerically for $k_{\perp}$. There are an infinite number of solutions to Eq. (14) and in keeping with the results from other experiments, ${ }^{12,13}$ we will assume that the lowest radial mode, the first root, is the dominant radial mode.

Based on the measured densities and applied magnetic field strength, the calculated parallel wavelength as a function of driving frequency is shown in Fig. 10 for 800 G. The wavelength is relatively constant in the low frequency range, but it increases significantly with increasing driving frequency above $11 \mathrm{MHz}$. That the wavelength increases with increasing frequency is perfectly acceptable for a bounded electromagnetic wave and arises from an ever increasing phase velocity. The calculated phase velocity as a function of driving frequency is shown in Fig. 11 for both 800 and 1000 G. 


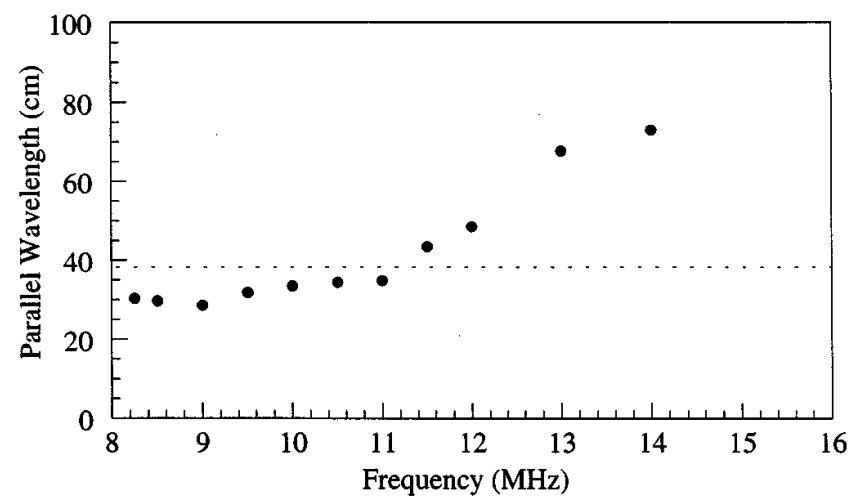

FIG. 10. Calculated parallel wavelength versus driving frequency at $800 \mathrm{G}$ and an rf power of $1 \mathrm{~kW}$. Dashed line indicates expected wavelength due to half-wavelength antenna.

Assuming that Landau damping is responsible for the energizing plasma electrons, the calculated phase velocities correspond to resonant electron energies $\left(E_{\mathrm{res}}=0.5 m_{e} v_{p}^{2}\right)$. The dependence of the measured density on resonant electron energy is shown in Fig. 12(a) for 600, 800, and $1000 \mathrm{G}$. The surprising aspect of Fig. 12(a) is that the density continues to increase as the resonant energy falls well below $50 \mathrm{eV}$, [consistent with the previous calculation based on Eq. (2)]. These more detailed calculations also imply Landau damping is not a viable candidate for electron energization in helicon sources. However, as mentioned previously, the calculations up to this point have used the chamber radius for the value of $a$ and visual observations suggest a distinct inner boundary to the plasma $(a \approx 5 \mathrm{~cm})$. If Eq. (14) is solved assuming a smaller plasma radius, the calculated phase velocities change significantly. Figure 12(b) is the same as Fig. 12(a) except that a smaller plasma radius $(a=5 \mathrm{~cm})$ is used in the calculations. The 800 and $1000 \mathrm{G}$ data follow a consistent trend of increasing density as the resonant energy nears $50 \mathrm{eV}$ (decreasing frequency). An even smaller plasma radius of $a$ $=3.5 \mathrm{~cm}$ is required to shift the $600 \mathrm{G}$ data so that the electron density peaks at a resonant energy of $50 \mathrm{eV}$ (the plasma core does appear narrower at lower magnetic field strengths). As the calculated resonant energy nears $50 \mathrm{eV}$ (decreasing driving frequency) the threshold power for initiation of the helicon mode also decreases (Fig. 13)—indicating improving ionization efficiency.

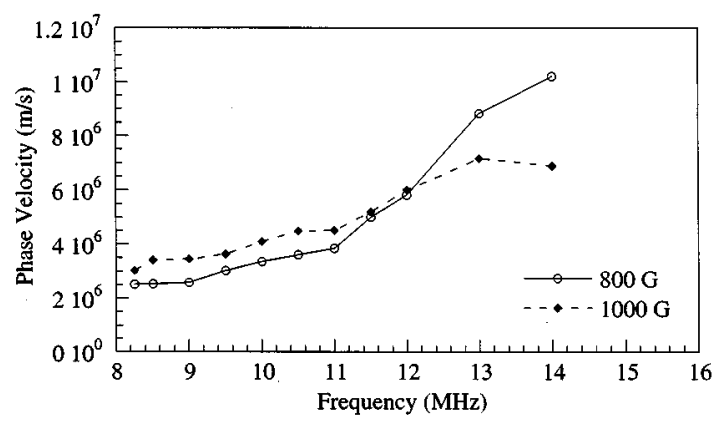

FIG. 11. Calculated phase velocity versus driving frequency for 800 and $1000 \mathrm{G}$ at an rf power of $1 \mathrm{~kW}$.
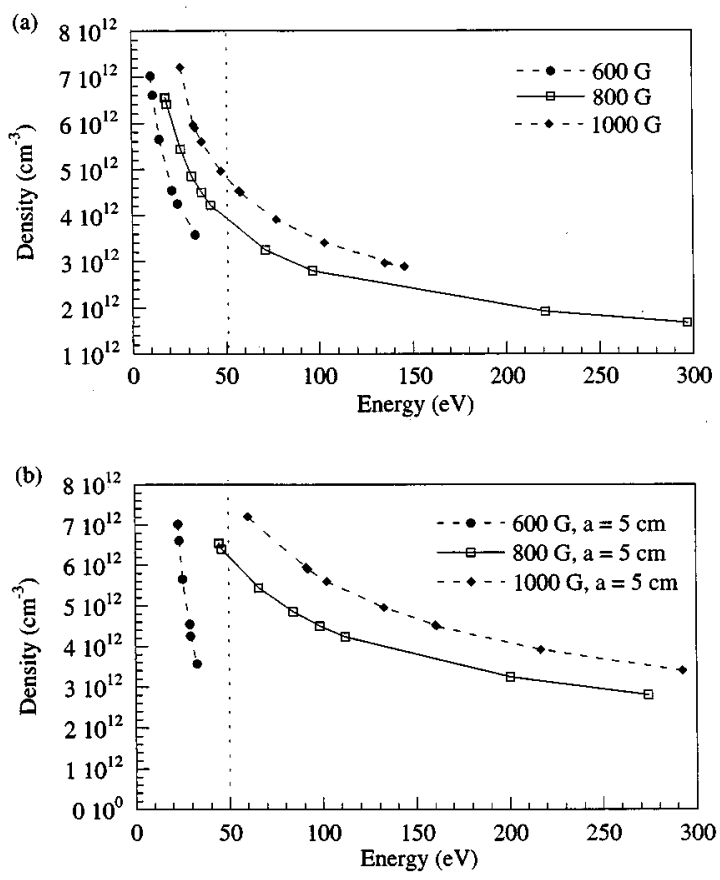

FIG. 12. (a) Electron density for three different magnetic field strengths versus calculated resonant electron energy assuming a plasma radius of 7.56 $\mathrm{cm}$. High energy $600 \mathrm{G}$ data not used as experiment were no longer in helicon mode. Dashed line marks $50 \mathrm{eV}$, energy for peak of electron impact ionization cross section. (b) Electron density for three different magnetic field strengths versus calculated resonant electron energy assuming a smaller plasma radius $(a=5 \mathrm{~cm})$.

If the densities in Fig. 12(b) started to decrease below $E_{\text {res }}$ of $50 \mathrm{eV}$, using a smaller plasma radius would be appropriate in the context of the Landau damping hypothesis. However, we were unable to find operating conditions for which the predicted $E_{\text {res }}$ was below $50 \mathrm{eV}$ and the density decreased. This was due to the fact that we lowered $E_{\text {res }}$ by decreasing the frequency which then yielded higher densities. Although it appears that Landau damping is not a viable candidate for the ionization efficiency of helicon sources, we do not believe that the data are unequivocal as a more localized radial mode structure is consistent with visual observations of the plasma. After reaching the helicon mode the radius of the blue core, due to Ar II emission, grows with increasing magnetic field.

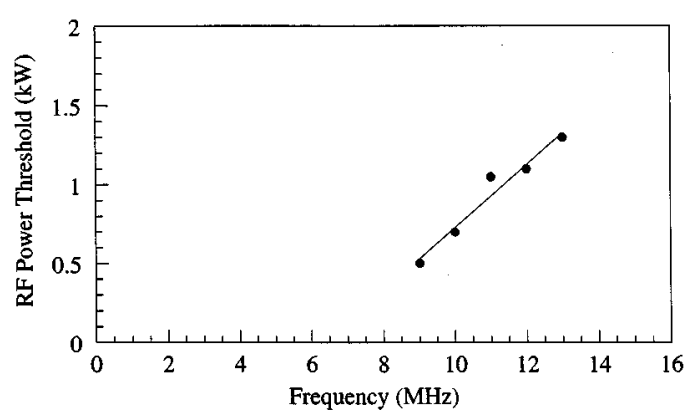

FIG. 13. Helicon rf power threshold versus driving frequency at a magnetic field of $600 \mathrm{G}$. Power threshold increases linearly with driving frequency. 


\section{SUMMARY}

By varying the driving frequency of a helicon source it is possible to maximize the plasma density. Not only does the density increase, but the power needed to launch the helicon mode can be reduced to a few hundred watts by optimizing the frequency for the applied magnetic field and the geometry of the experiment. These measurements suggest two possible interpretations. If we assume the boundary for the helicon wave is the chamber radius, then Landau damping cannot be responsible for the highly efficient ionization in helicon sources. If we assume that Landau damping must be responsible for the effective ionization, we are forced to conclude that the boundary for the helicon wave is not the chamber wall. Measurements of the wave phase velocity at various frequencies in our experiment will provide enough information to determine which interpretation is correct. Measurements of $k_{\|}$and $k_{\perp}$ vs $f$ are underway and the results will be reported in a future work. In particular, the plasma density as a function of wave resonant energies above and below $50 \mathrm{eV}$ [e.g., Fig. 12(b)] will provide critical information about the energy deposition process in helicon sources.

For the purpose of discussion, let us assume that Landau does play a role in the ionization process and therefore the parallel phase velocity should be designed to correspond to a $50 \mathrm{eV}$ resonant energy. Equation (14) predicts that a driving frequency of $f=2.0 \mathrm{MHz}$ is needed to generate a helicon plasma of density $1 \times 10^{13} \mathrm{~cm}^{-3}$ across the entire plasma column $(a=7.56 \mathrm{~cm})$ for a magnetic field of $1 \mathrm{kG}$. To achieve a higher density of $5 \times 10^{13} \mathrm{~cm}^{-3}$, a lower frequency of $f=1.3 \mathrm{MHz}$ is needed. It is worth noting that $1.3 \mathrm{MHz}$ is well below the lower hybrid frequency for a $1000 \mathrm{G}$ magnetic field $\left(f_{\mathrm{LH}}=10 \mathrm{MHz}\right)$. This suggests that the frequency needed to generate large volume, fully helicon plasmas lies outside of the range typically used for helicon experiments. Our preliminary helicon experiments in helium at a driving frequency of $10 \mathrm{MHz}$ and a magnetic field of $800 \mathrm{G}$ $\left(f_{\mathrm{LH}}=28 \mathrm{MHz}\right)$ suggest that is it possible to operate helicon sources well below the lower hybrid frequency, and thus very high helicon densities at low rf power may be possible.

Another consideration in the discussion of Landau damping is the Landau damping rate. If we compare the maximum Landau damping rate $(\text { at } \lambda \cong 18.8 \mathrm{~cm})^{9,23}$

$$
\begin{aligned}
{\left[\frac{\operatorname{Im}(k)}{\operatorname{Re}(k)}\right]_{\mathrm{LD}} } & =2 \sqrt{\pi} \frac{v_{t h}}{\omega_{c e}} \frac{3.83}{a}\left(\frac{\omega}{k v_{t h}}\right)^{4} \exp \left(-\left(\frac{\omega}{k v_{t h}}\right)^{2}\right) \\
& \approx 7 \times 10^{-3}
\end{aligned}
$$

to the collisional damping rate ${ }^{9,23}$ for our typical parameters,

$$
\left[\frac{\operatorname{Im}(k)}{\operatorname{Re}(k)}\right]_{\mathrm{CD}}=\frac{\nu_{e i}}{\omega} \frac{c^{2}}{\omega_{p e}^{2}}\left(\frac{3.83}{a}\right)^{2} \approx 2 \times 10^{-3},
$$

we find that although the Landau damping rate is extremely small, it is still larger than the collisional damping rate. Recently Degeling et al. ${ }^{9}$ suggested a particle trapping model that predicts that the plasma density should increase exponentially with increasing rf power when the phase velocity of the wave corresponds to a $50 \mathrm{eV}$ resonant energy. In contrast, the $9 \mathrm{MHz}$ data shown in Fig. 7, for which the resonant energy should be close to $50 \mathrm{eV}$, are relatively insensitive to rf power once the helicon mode is reached. For our magnetic fields and densities, cyclotron damping ${ }^{19}$ is not expected to play a significant role.

\section{ACKNOWLEDGMENTS}

This work was supported by funds provided by West Virginia University (WVU). We would like to thank Carl Weber and Tom Milam of our instrument shop for an excellent job of fabricating the experimental apparatus, the plasma physics group at the University of Wisconsin-Madison for the loan of critically needed hardware, the Q-machine group at WVU for technical support and useful scientific discussions, and Jim Gilland at UW-Madison for probe construction advice.

${ }^{1}$ R. W. Boswell, Phys. Lett. A 33, 457 (1970).

${ }^{2}$ R. W. Boswell, Plasma Phys. Controlled Fusion 26, 1147 (1984).

${ }^{3}$ R. Boswell, R. Porteous, A. Prytz, A. Bouchoule, and P. Ranson, Phys. Lett. A 91, 163 (1982).

${ }^{4}$ F. F. Chen, J. Vac. Sci. Technol. A 10, 1389 (1992).

${ }^{5}$ F. F. Chen and C. D. Decker, Plasma Phys. Controlled Fusion 34, 635 (1992).

${ }^{6}$ G. Chevalier and F. F. Chen, J. Vac. Sci. Technol. A 11, 1165 (1993).

${ }^{7}$ A. W. Degeling, C. O. Jung, R. W. Boswell, and A. R. Ellingboe, Phys. Plasmas 3, 2788 (1996).

${ }^{8}$ A. R. Ellingboe and R. W. Boswell, Phys. Plasmas 3, 2797 (1996).

${ }^{9}$ A. Komori, T. Shoji, K. Miyamoto, J. Kawai, and Y. Kawai, Phys. Fluids B 3893 (1991).

${ }^{10}$ T. Shoji, Y. Sakawa, S. Nakazawa, K. Kadota, and T. Sato, Plasma Sources Sci. Technol. 2, 5 (1993).

${ }^{11}$ I. D. Sudit and F. F. Chen, Plasma Sources Sci. Technol. 5, 43 (1996).

${ }^{12}$ R. T. S. Chen, R. A. Breun, S. Gross, N. Hershkowitz, M.-K. J. Hseih, and J. Jacobs, Plasma Sources Sci. Technol. 4, 337 (1995).

${ }^{13}$ M. Light and F. F. Chen, Phys. Plasmas 2, 1084 (1995).

${ }^{14}$ M. Light, I. D. Sudit, F. F. Chen, and D. Arnush, Phys. Plasmas 2, 4094 (1995).

${ }^{15}$ J. P. Klosenberg, B. McNamara, and P. C. Thonemann, J. Fluid Mech. 21, 545 (1965).

${ }^{16}$ F. F. Chen, M. J. Hsieh, and M. Light, Plasma Sources Sci. Technol. 3, 49 (1994).

${ }^{17}$ F. F. Chen, Plasma Phys. Controlled Fusion 33, 339 (1991).

${ }^{18}$ S. Okamura, K. Adati, T. Aoki, D. R. Baker, H. Fujita, H. R. Garner, K. Hattori, S. Hidekuma, T. Kawamoto, R. Kumazawa, Y. Okubo, and T. Sato, Nucl. Fusion 26, 1491 (1986).

${ }^{19}$ B. M. Harvey and C. N. Lashmore-Davies, Phys. Fluids B 5, 3864 (1993).

${ }^{20}$ I. D. Sudit and F. F. Chen, Plasma Sources Sci. Technol. 3, 162 (1994).

${ }^{21}$ C. Laviron, A. J. H. Donne, M. E. Manso, and J. Sanchez, Plasma Phys. Controlled Fusion 38, 905 (1996).

${ }^{22}$ J-H. Kim and H-Y. Chang, Phys. Plasmas 3, 1462 (1996).

${ }^{23}$ F. F. Chen, in Proceedings of the Second International Conference on Plasma Physics, Kiev, 1987, edited by A. G. Sitenko (Naukova Dumka, Kiev, 1987), Vol. 4, p. 321. 